\title{
Optimal Power Allocation Strategy Against Jamming Attacks Using the Colonel Blotto Game
}

\author{
Yongle Wu, Beibei Wang, and K. J. Ray Liu \\ Department of Electrical and Computer Engineering, \\ University of Maryland, College Park, MD 20742, USA. \\ \{wuyl, bebewang, kjrliu\}@umd.edu
}

\begin{abstract}
Cognitive radio technologies have become a promising approach to increase the efficiency of spectrum utilization. Although cognitive radio has been intensively studied in recent years, only a few works have discussed security aspects. In this paper, we focus on the jamming attack, one of major threats to cognitive radio networks, where a malicious user wants to jam the communications of secondary users by injecting interference. Aware of the absence of several primary users and the presence of a malicious user, a secondary user can allocate power to those fallow bands with a randomized strategy, in hope of alleviating the damage caused by the malicious user. We model this scenario into a two-player zero-sum game, and derive its unique Nash Equilibrium under certain conditions using the Colonel Blotto game approach, which provides a minimax strategy that the secondary user should adopt in order to minimize the worstcase damage caused by the malicious user. Simulation results are presented to verify the performance.
\end{abstract}

\section{INTRODUCTION}

As the last decade witnessed a dramatic increase in the demand for radio spectrum, cognitive radio [1] has become a promising technology, opening up a new paradigm where unlicensed users (secondary users) are allowed to access licensed bands on a non-interference basis to legacy spectrum holders (primary users). Cognitive radio technologies have been intensively studied in recent years. For example, spectrum sensing techniques were investigated in [2], and [3] showed that the sensing time could be reduced and spectrum agility could be enhanced through user cooperation. In [4], the noregret learning algorithm was employed to improve the performance of opportunistic spectrum access in a fully distributive cognitive radio network. Moreover, dynamic spectrum access could also be implemented on a pricing/negotiation basis when secondary users lease short-term spectrum rights from primary users, such as a real-time auction in [5], and a double auction in [6]. In [7], a multi-winner auction was formed according to the interference-limited nature of spectrum resources, and auction mechanisms have been carefully designed to eliminate all possible kinds of collusion.

However, most of existing works have not taken security issues into consideration, which may lead to a severe loss when a malicious user shows up. A malicious user, who may be an enemy in the context of military communications or a business rival in the context of civilian communications, aims at maximizing the damage that he/she causes. In fact, cognitive radio networks are extremely vulnerable to malicious attacks, partly because secondary users do not own the spectrum, and hence their opportunistic access cannot be protected from adversaries. So far, there have been only a few papers regarding security issues. For instance, the primary user emulation attack was described and a transmitter verification scheme was proposed to test whether the given signal came from a primary user in [8]; [9] employed a Hammer model to identify, analyze and assess denial of service attacks; in [10], security issues of the IEEE 802.22 standard were addressed; [11] discussed an attack that malicious users attempted to affect an artificialintelligence-based secondary user's behavior by misleading the learning process.

In this paper, we mainly focus on one kind of malicious attacks in cognitive radio networks, namely, the jamming attack [12], where a malicious user, or the "jammer", wants to prevent or jam the communications of a secondary user. The secondary user, on the other hand, monitors the presence/absence of several primary users to find spectrum opportunities, and allocates power to those fallow bands using some randomized strategy in order to avoid the jamming as much as possible. We investigate the scenario with one secondary user and one jammer, and model their interaction into the Colonel Blotto game which originates to solve the problem where two players simultaneously distribute forces across multiple battlefields [13]. We want to know how much damage the jammer can cause to the secondary user, and what strategy the secondary user should adopt. Moreover, the jammer may be able to gradually accumulate some knowledge about the secondary user's strategy, and adjust his/her own strategy to maximize damage given the secondary user's strategy. Therefore, the secondary user should adopt a strategy such that the corresponding worst-case damage is minimized. We derive the unique Nash Equilibrium (NE) of the jamming game under some conditions. Moreover, we show that the NE strategy is optimal in the sense that it minimizes the worst-case damage caused by the jammer.

The remainder of this paper is organized as follows. In Section II, the interaction between a secondary user and a jammer is modeled into a two-player zero-sum game. In Section III, we modify the game into a Colonel Blotto game, and derive the NE of the game, that is, the optimal strategy of power allocation. Simulation results are presented in section IV, followed by Section V that concludes this paper.

\section{SySTEM MODEL}

Consider the case when a secondary user, equipped with multiple radios, wants to transmit several packets to its receiver 
using a total amount of power $P$. This also applies to the scenario where multiple secondary users cooperate to fight against the jammer and hence can be regarded as a single "super" user. At the beginning of each time slot, the secondary user senses a number of bands to find the fallow ones that are not occupied by primary users. When there are $M$ fallow bands available, the user allocates power $p_{k}$ to the $k$ th band, under the constraint that the total power should not exceed the limit $\sum_{k=1}^{M} p_{k}=P$. At the same time, a jammer wants to sabotage on the communication link by injecting interference power $i_{k}$ to the $k$ th band. We assume the malicious user also has a total power constraint $\sum_{k=1}^{M} i_{k}=I$, where $I$ is the power budget. Without loss of generality, we assume all channel gains are 1, since they can be absorbed into the power term otherwise. At the receiver, if the received signalto-interference-and-noise ratio (SINR) exceeds the minimum requirement $\beta$ ( $\beta$ is determined by the type of service), i.e.,

$$
\frac{p_{k}}{i_{k}+N_{k}} \geq \beta
$$

packets can be transmitted successfully; otherwise, the link is too poor to be useful. $N_{k}$ is the variance of white noise when the receiver receives signal from channel $k$.

In the presence of a jammer, the secondary user wants to maximize the number of successful transmissions, by randomizing his/her power allocation strategy. On the other hand, the jammer also wants to find a randomized power allocation strategy such that the number of the secondary user's successful transmissions is minimized. Assume both the secondary user and the jammer have perfect spectrum sensing that they know the number of fallow bands $M$ at each time slot. Then, the secondary user and the jammer play a game in every time slot, which is defined as follows and illustrated in Fig. 1.

- Players: there are two players in this game. One is the secondary user, and the other is the jammer.

- Actions: both players allocate power to each band as $\left(p_{1}, p_{2}, \ldots, p_{M}\right)$ and $\left(i_{1}, i_{2}, \ldots, i_{M}\right)$. Their mixed strategies are described by probability distribution functions $F\left(p_{1}, p_{2}, \ldots, p_{M}\right)$ and $H\left(i_{1}, i_{2}, \ldots, i_{M}\right)$, respectively.

- Payoffs: after both players decide their power allocation, the secondary user's payoff is given by the number of successful transmissions, i.e., $\sum_{k=1}^{M} \mathbf{1}\left(\frac{p_{k}}{i_{k}+N_{k}} \geq \beta\right)$ where $\mathbf{1}(\cdot)$ is the indicator function, returning 1 when the argument in the parenthesis holds true and 0 otherwise, and the jammer's payoff is the opposite, i.e., $-\sum_{k=1}^{M} \mathbf{1}\left(\frac{p_{k}}{i_{k}+N_{k}} \geq \beta\right)$. This is a zero-sum game because payoffs of two players always add up to zero.

Assuming both players have full information including $P, I$, $N$ and $\beta$, we will find the NE for this game. If the secondary user's payoff is defined as $s(F, H)$ when players choose $F$ and $H$ as their strategies, the NE strategy $\left(F^{*}, H^{*}\right)$ is such a pair that $s\left(F, H^{*}\right) \leq s\left(F^{*}, H^{*}\right)$ for any $F \neq F^{*}$ and $-s\left(F^{*}, H\right) \leq-s\left(F^{*}, H^{*}\right)$ for any $H \neq H^{*}$. Because this is a zero-sum game, the NE not only provides an equilibrium point from which neither players have the incentive to deviate unilaterally, but also is a minimax strategy [14] such that

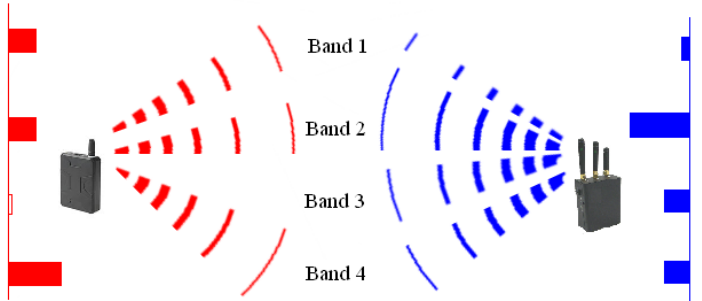

Fig. 1. Illustration of a jamming game where a secondary user (left) aims at maximizing the number of successful transmissions by using a randomized strategy to allocate power in four bands and a jammer (right) attempts to block those transmissions by injecting interference.

$s\left(F^{*}, H^{*}\right) \geq \min _{H} s(F, H)$ for any other strategy $F$. This property is very important. If the jammer is capable of learning the secondary user's strategy $F$, he/she can always come up with a strategy $H$ targeted at the specific strategy $F$ such that the damage is maximized. Among all strategies, $F^{*}$ is the optimal in the sense that the worst-case damage is minimized.

\section{NASh EQUilibrium OF THE GAmE}

In this section, we will find the Nash Equilibrium for the proposed jamming game by modeling it to a Colonel Blotto game where two opponents distribute limited resources over a number of battlefields with a payoff equal to the sum of outcomes from individual battlefield [13]. To this end, we define $j_{k}=\beta\left(i_{k}+N_{k}\right)$, and assume that in the new game the jammer allocates $j_{k}$ to the $k$ th band under a total power constraint $\sum_{k=1}^{M} j_{k}=\beta\left(I+\sum_{k=1}^{M} N_{k}\right) \triangleq J$. Different from the traditional Colonel Blotto game where there is no minimum constraint on resource allocated, in this jamming game, $j_{k}$ has to be lower bounded by $\beta N_{k}$, since in fact the jammer only has control over $i_{k}$, and $j_{k} \geq \beta N_{k}$ with equality when $i_{k}=0$. In this new game, the jammer's strategy is also given by a joint distribution function, denoted by $G\left(j_{1}, j_{2}, \ldots, j_{M}\right)$, whose marginal distribution functions are given by $G_{1}\left(j_{1}\right), G_{2}\left(j_{2}\right), \ldots, G_{M}\left(j_{M}\right)$. Similarly, the marginal distribution functions of the secondary user's power allocation are denoted by $F_{1}\left(p_{1}\right), F_{2}\left(p_{2}\right), \ldots, F_{M}\left(p_{M}\right)$.

Notice that the probability of a successful transmission is $\operatorname{Pr}\left(\frac{p_{k}}{i_{k}+N_{k}} \geq \beta\right)=\operatorname{Pr}\left(j_{k} \leq p_{k}\right)=G_{k}\left(p_{k}\right)$, the payoff of the secondary user is $\sum_{k=1}^{M} G_{k}\left(p_{k}\right)$ when he/she fixes the power allocation as $\left(p_{1}, p_{2}, \ldots, p_{M}\right)$. When the player employs a randomized strategy, the expected payoff becomes

$$
\sum_{k=1}^{M} \int_{0}^{\infty} G_{k}\left(p_{k}\right) \mathrm{d} F_{k}\left(p_{k}\right),
$$

and the necessary condition of the total power constraint becomes

$$
P=E\left(\sum_{k=1}^{M} p_{k}\right)=\sum_{k=1}^{M} \int_{0}^{\infty} p_{k} \mathrm{~d} F_{k}\left(p_{k}\right) .
$$

If we introduce a Lagrangian multiplier $\lambda_{P}$, the optimization problem of the secondary user can be formulated as

$$
\max _{\left\{F_{k}\left(p_{k}\right)\right\}} \sum_{k=1}^{M} \int_{0}^{\infty}\left(G_{k}\left(p_{k}\right)-\lambda_{P} p_{k}\right) \mathrm{d} F_{k}\left(p_{k}\right)+\lambda_{P} P .
$$


Similarly, we can derive the optimization problem for the jammer who attempts to maximize $\sum_{k=1}^{M} \mathbf{1}\left(p_{k}<j_{k}\right)$. As shown later in this section, the distribution function $F_{k}\left(p_{k}\right)$ has no discontinuous point except $p_{k}=0$, which means the amount of power allocated in the $k$ th channel is a random variable with a discrete part at 0 and a continuous part elsewhere. Hence, the event $p_{k}=j_{k}$ happens with probability 0 , and $\operatorname{Pr}\left(p_{k}<j_{k}\right)=\operatorname{Pr}\left(p_{k} \leq j_{k}\right)=F_{k}\left(j_{k}\right)$. Therefore, from the jammer's point of view, the optimization problem is

$$
\max _{\left\{G_{k}\left(j_{k}\right)\right\}} \sum_{k=1}^{M} \int_{\beta N_{k}}^{\infty}\left(F_{k}\left(j_{k}\right)-\lambda_{J} j_{k}\right) \mathrm{d} G_{k}\left(j_{k}\right)+\lambda_{J} J,
$$

where $\lambda_{J}$ is the Lagrangian multiplier for the jammer.

For the secondary user, he/she can either decide not to access band $k$ (i.e., $p_{k}=0$ ) or decide to access that channel with some power lower bounded by $\underline{p}_{k}$ and upper bounded by $\bar{p}_{k}$ (i.e., $p_{k} \in\left[\underline{p}_{k}, \bar{p}_{k}\right]$ ). Apparently, $\underline{p}_{k} \geq \beta N_{k}$, because if $p_{k}$ is chosen in the open interval $\left(0, \beta N_{k}\right)$, the secondary user will always fail in that band, and it is better not to allocate power at all. When the equilibrium strategy is a mixed strategy over the domain $0 \cup\left[p_{k}, \bar{p}_{k}\right]$, according to game theory, the player must be indifferent among these values [14], namely, $G_{k}\left(p_{k}\right)-\lambda_{P} p_{k}=$ constant for $p \in 0 \cup\left[\underline{p}_{k}, \bar{p}_{k}\right]$. In particular, since $G_{k}(0)=0$, we can further have

$$
G_{k}\left(p_{k}\right)-\lambda_{P} p_{k}=0 \text {, for } p_{k} \in 0 \cup\left[\underline{p}_{k}, \bar{p}_{k}\right] \text {. }
$$

The similar argument can be applied to the jammer who allocates power $j_{k} \in\left[j_{k}, \bar{j}_{k}\right]$ and has to be indifferent among the values, namely,

$$
F_{k}\left(j_{k}\right)-\lambda_{J} j_{k}=\text { constant, for } j_{k} \in\left[\underline{j}_{k}, \bar{j}_{k}\right] \text {. }
$$

For convenience, we will assume $N_{1}=N_{2}=\ldots=N_{M}=$ $N$ in the following, but our results can be extended to cases when variances of noise are different.

Lemma 1: For the NE strategy, bounds are determined as $\bar{p}_{k}=\bar{j}_{k}=\min \left(1 / \lambda_{P}, 1 / \lambda_{J}\right)$, and $\underline{p}_{k}=\underline{j}_{k}=\beta N$. Moreover, $\operatorname{Pr}\left(j_{k}=\beta N\right)=\lambda_{P} \beta N$, and $\operatorname{P} r\left(p_{k}{ }^{k}=\beta N\right)=0$; the probability distribution function $F_{k}\left(p_{k}\right)$ is continuous in the range $\left(\beta N, \bar{p}_{k}\right]$, and so is $G_{k}\left(j_{k}\right)$.

Proof: According to the definition of the NE, no single player can be better off by deviating unilaterally from the NE strategy. In what follows, we give a proof mainly by contradiction.

From optimization problems (4) and (5), it is clear that $p_{k} \leq$ $1 / \lambda_{P}$ and $j_{k} \leq 1 / \lambda_{J}$ have to be satisfied to avoid negative payoffs. $\bar{p}_{k}=\overline{\bar{j}}_{k}$ can be proved by contradiction. If $\bar{p}_{k} \neq \bar{j}_{k}$, say $\bar{p}_{k}<\bar{j}_{k}$, the jammer is better off by moving $\bar{j}_{k}$ to $\left(\bar{p}_{k}+\right.$ $\left.\bar{j}_{k}\right) / 2$, as $F_{k}\left(\bar{j}_{k}\right)-\lambda_{J} \bar{j}_{k}=1-\lambda_{J} \bar{j}_{k}<1-\lambda_{J}\left(\bar{p}_{k}+\bar{j}_{k}\right) / 2=$ $F_{k}\left(\left(\bar{p}_{k}+\bar{j}_{k}\right) / 2\right)-\lambda_{J}\left(\bar{p}_{k}+\bar{j}_{k}\right) / 2$. The analysis is similar for the case $\bar{p}_{k}>\bar{j}_{k}$.

Next, we prove $\underline{p}_{k}=\underline{j}_{k}$ by contradiction. If $\underline{p}_{k} \neq \underline{j}_{k}$, say $\underline{p}_{k}<\underline{j}_{k}$, the secondary user is better off by moving $\left(\underline{p}_{k}+\underline{j}_{k}\right) / 2$ to $\underline{p}_{k}$, since power can be saved without affecting the winning probability. The analysis is similar for the case $p_{k}>j_{k}$.

According to (6), $G\left(\underline{j}_{k}\right)=\lambda_{P} \underline{j}_{k}$, and hence $\operatorname{Pr}\left(j_{k}=\underline{j}_{k}\right)=$ $\lambda_{P} \underline{j}_{k}$. Because $p_{k} \geq \underline{j}_{k}$ always holds for $p_{k} \in\left[\underline{p}_{k}, \bar{p}_{k}\right]$, by contradiction, if $\underline{j}_{k}>\beta N$, the jammer will be better off by moving $\underline{j}_{k}$ to $\beta \bar{N}$. Therefore, $\underline{p}_{k}=\underline{j}_{k}=\beta N$.
Then, if $\operatorname{Pr}\left(p_{k}=\beta N\right)>0$, the jammer can change the probability mass from $\beta N$ to $\beta N+\epsilon$ where $\epsilon$ is an arbitrary small number, and can increase the jamming probability by $\lambda_{P} \beta N \cdot \operatorname{Pr}\left(p_{k}=\beta N\right)$ with only negligible power increase. This cannot be an NE, and as a result, $\operatorname{Pr}\left(p_{k}=\beta N\right)=0$.

Finally, we show that $F_{k}\left(p_{k}\right)$ cannot have discontinuous points in the interval $\left(\beta N, \bar{p}_{k}\right]$. By contradiction, assume there is at least one discontinuous point, denoted by $p^{o}$, and thus $\operatorname{Pr}\left(p_{k}=p^{o}\right)>0$. Then, the jammer can move the neighborhood $\left(p^{o}-\epsilon, p^{o}\right)$ to $\left(p^{o}, p^{o}+\epsilon\right)$ to increase the jamming probability by $\operatorname{Pr}\left(p_{k}=p^{o}\right) \cdot \operatorname{Pr}\left(j_{k} \in\left(p^{o}-\epsilon, p^{o}\right)\right)$ with only negligible power increase when $\epsilon$ is an arbitrary small number. Similar arguments can be made to prove $G_{k}\left(j_{k}\right)$ cannot have discontinuous points in the interval $\left(\beta N, \bar{j}_{k}\right]$ either.

Lemma 1 presents several basic properties of marginal distribution functions, which must be possessed by the NE strategy. Based on the lemma and other analysis we have made above, the NE of the jamming game can be fully characterized by the following theorem.

Theorem 1: When parameters satisfy the condition $P \leq$ $\beta I \sqrt{1+2 M N / I}$, a unique NE exists, and the marginal distribution of the NE strategy is given by

$$
F_{k}\left(p_{k}\right)=\left\{\begin{array}{cc}
0, & p_{k}<0, \\
1-\lambda_{J} / \lambda_{P}+\lambda_{J} \beta N, & p_{k} \in[0, \beta N), \\
1-\lambda_{J} / \lambda_{P}+\lambda_{J} p_{k}, & p_{k} \in\left[\beta N, 1 / \lambda_{P}\right],
\end{array}\right.
$$

for the secondary user, and

$$
H_{k}\left(i_{k}\right)=\left\{\begin{array}{cc}
0, & i_{k}<0, \\
\lambda_{P} \beta\left(N+i_{k}\right), & i_{k} \in\left[0,1 /\left(\beta \lambda_{P}\right)-N\right],
\end{array}\right.
$$

for the jammer, where $\lambda_{P}=M /\left(J+\sqrt{J^{2}-\beta^{2} M^{2} N^{2}}\right)$ and $\lambda_{J}=M P /\left(J^{2}-\beta^{2} M^{2} N^{2}+J \sqrt{J^{2}-\beta^{2} M^{2} N^{2}}\right)$.

Proof: Since $\bar{p}_{k}=\bar{j}_{k}=\min \left(1 / \lambda_{P}, 1 / \lambda_{J}\right)$ are independent on $k$, we define $\bar{p} \triangleq \bar{p}_{k}=\bar{j}_{k}$. According to Lemma 1 , $F_{k}\left(p_{k}\right)$ is continuous in the interval $[\beta N, \bar{p}]$, and therefore, we can take the derivative of (7), and plug the result

$$
\mathrm{d} F_{k}(x)=\lambda_{J} \mathrm{~d} x, x \in[\beta N, \bar{p}]
$$

to the constraint given by equation (3):

$$
P=\sum_{k=1}^{M} \int_{0}^{\bar{p}} p_{k} \mathrm{~d} F_{k}\left(p_{k}\right)=0+M \int_{\beta N}^{\bar{p}} \lambda_{J} p_{k} \mathrm{~d} p_{k}=\frac{M}{2} \lambda_{J}\left(\bar{p}^{2}-\beta^{2} N^{2}\right) \text {. }
$$

Similar derivation can be done to the jammer's power constraint as follows with special care, because $G_{k}\left(j_{k}\right)$ is discontinuous at $j_{k}=\beta N$,

$$
J=M\left(\beta N\left(\lambda_{P} \beta N\right)+\frac{1}{2} \lambda_{P}\left(\bar{p}^{2}-\beta^{2} N^{2}\right)\right) .
$$

If $1 / \lambda_{P} \leq 1 / \lambda_{J}$, then $\bar{p}=1 / \lambda_{P}$ and (12) becomes a quadratic equation of the variable $1 / \lambda_{P}$, two roots of which are given by

$$
\left(\frac{1}{\lambda_{P}}\right)_{1,2}=\frac{1}{M}\left(J \pm \sqrt{J^{2}-\beta^{2} M^{2} N^{2}}\right) .
$$

However, only the root with the plus sign is valid since the other root is smaller than $\beta N$. Then, $1 / \lambda_{J}$ can be solved from (11) accordingly,

$$
\frac{1}{\lambda_{J}}=\frac{J^{2}-\beta^{2} M^{2} N^{2}+J \sqrt{J^{2}-\beta^{2} M^{2} N^{2}}}{M P} .
$$


The condition $1 / \lambda_{P} \leq 1 / \lambda_{J}$ holds when $P \leq$ $\beta I \sqrt{1+2 M N / I} \approx \beta I$. Another pair of parameters can be solved under the situation that $1 / \lambda_{P}>1 / \lambda_{J}$, but we are more interested in the previous case where the jammer is more powerful.

Under the condition $P \leq \beta I \sqrt{1+2 M N / I}$, the pair of Lagrangian multipliers have been uniquely determined by (13) and (14). Since at least one mixed-strategy NE exists in a game [14], we can safely draw a conclusion that this characterizes the unique NE in the jamming game. Based on the derived parameters and (6) (7), it is straightforward to write down the marginal distribution of the NE strategy as in the theorem.

So far, we have known the existence of the NE and the formula of marginal distribution functions; however, it still remains a question to find the specific NE strategy determined by the joint probability distribution function. We have followed the procedure in [13] to construct one kind of joint distribution that matches desired marginal distribution and meets the total power restriction, and applied it in our simulation experiments. With this procedure, we can finally obtain the NE strategy for the jamming game.

\section{Simulation Results}

In this section, we present some simulation results to evaluate the proposed jamming game. We assume that the secondary user has a total power constraint $P=300 \mu \mathrm{W}$, the noise power in each band is $N=1 \mu W$, the jammer has a total power budget $I$, and the required SINR threshold is $\beta$.

First, we want to validate through simulation the procedure that generates a kind of joint distribution by using marginal distribution functions and the total power constraint. We have examined the output samples from the procedure under a variety of settings, and found the resultant empirical marginal distribution always matches the expected shape. For instance, when $I=30 \mu W, \beta=10 d B$, and $M=10$, the empirical marginal distribution from 500 samples has been plotted in Fig. 2, which is consistent with equation (8) in Theorem 1 for the secondary user and equation (9) for the jammer.

Next, we manipulate parameters of the system to gain an insight on how they affect the secondary user's equilibrium payoff, that is, the average number of successful transmissions per time slot. Fig. 3 shows that the payoff increases with the number of bands available when $\beta=10 \mathrm{~dB}$. This is because the secondary user has more freedom to randomly choose bands to allocate power when there are more bands. The payoffs versus the required SINR threshold $\beta$ with $M=10$ are shown in Fig. 4. Since $\beta$ actually leverages the effect of noise and interference, the more stringent requirement of the SINR is (i.e., the higher $\beta$ is), the smaller number of successful transmissions can be achieved. In addition, in both figures, jamming causes more damage when the jammer has a higher power budget.

In order to show that the NE strategy minimizes the worstcase damage for the secondary user, we have run simulations with two other possible strategies considered: one decides the number of bands to access according to the NE strategy
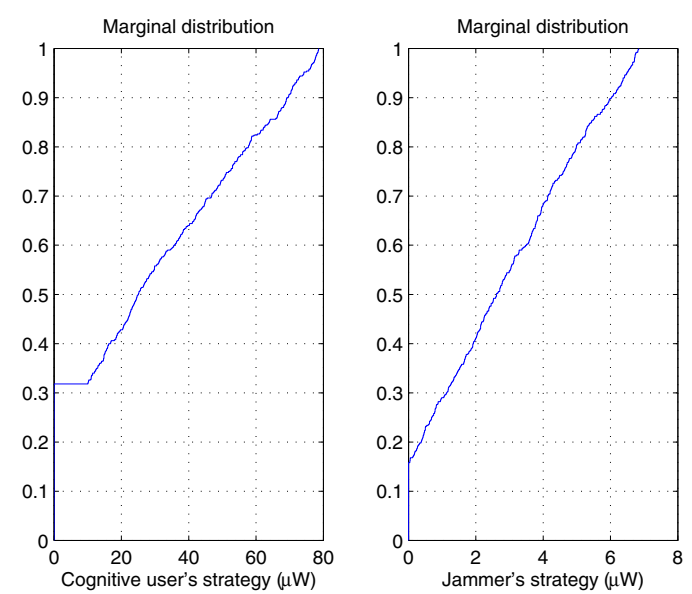

Fig. 2. Empirical marginal distribution of the joint distribution constructed from marginal distribution functions under total power constraints.

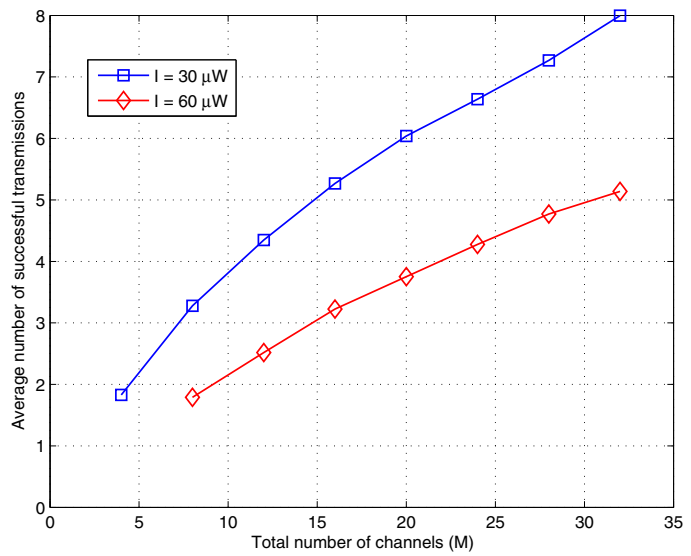

Fig. 3. The average number of successful transmissions when the number of bands increases.

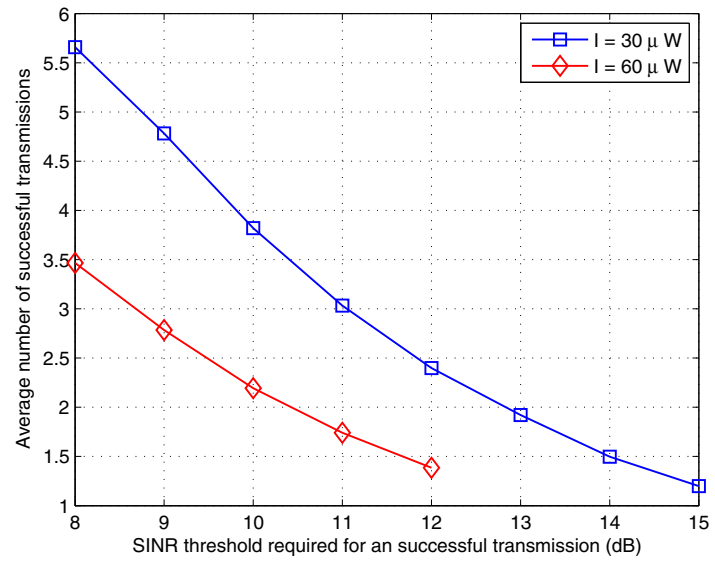

Fig. 4. The average number of successful transmissions when the required SINR threshold increases. 


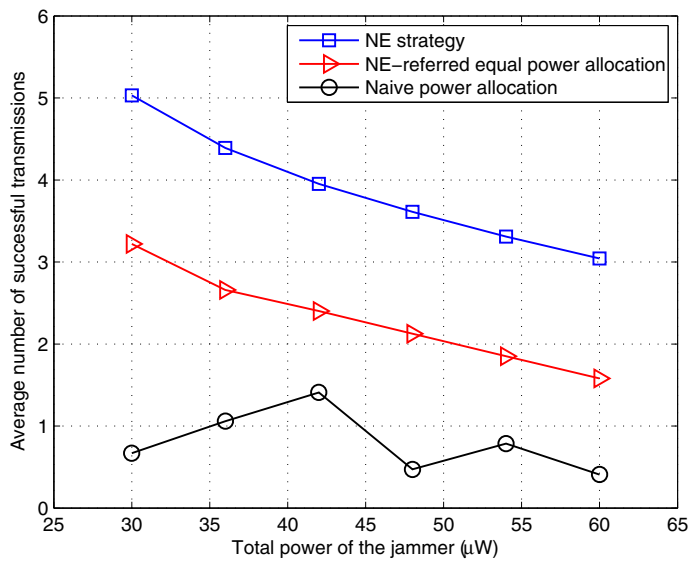

Fig. 5. The average number of successful transmissions when the secondary user employs different power allocation strategies and the jammer is able to adjust his/her jamming strategy.

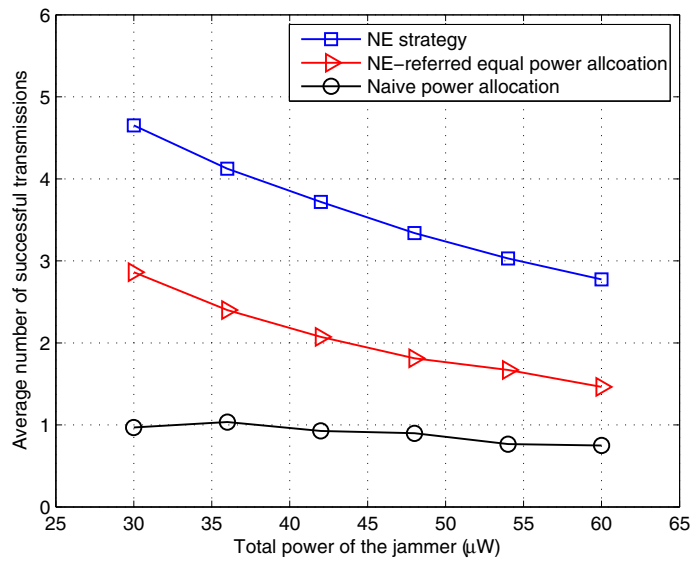

Fig. 6. The average number of successful transmissions with different strategies adopted by the secondary user, in the scenario where the number of fallow bands varies from time slot to time slot due to presence/absence of primary users.

but allocates power equally, and the other allocates power based on a naive assumption that the jammer would inject equal interference to each band. They are referred to as "NEreferred equal power allocation" and "naive power allocation", respectively. Fig. 5 provides the average number of successful transmissions with $\beta=10 \mathrm{~d} B, M=15$ and varying power budgets $I$ when the jammer is capable of adapting his/her strategy by learning the secondary user's behavior. From the figure, we can see the secondary user suffers from significant loss if using strategies other than the NE strategy. Therefore, the secondary user has to conform to the NE strategy, which guarantees a bounded damage caused by the jammer.

Finally, we take into consideration the access pattern of primary users and present the performance. Assume there are fifteen bands in total, each of which is independently occupied by its primary user with a probability of 0.1 . Fig. 6 provides the number of successful transmissions when the secondary user adopts the NE strategy, the "NE-referred equal power allocation" strategy, and the "naive power allocation" strategy, respectively. It is clear that the NE strategy performs much better than the other two strategies, and the secondary user has to choose it as the optimal power allocation strategy against malicious jamming attacks.

\section{CONCLUSIONS}

In this paper, we have modeled the power allocation problem under malicious jamming attacks as a two-player zerosum game where the secondary user aims at maximizing the number of successful transmissions by randomizing amounts of power allocated to each band. This jamming game has been converted to a Colonel Blotto game, the unique Nash Equilibrium of which can be derived under certain conditions. Since the NE strategy of a zero-sum game is also a minimax strategy, power allocation using the NE is optimal which minimizes the worst-case damage caused by the jammer. Finally, simulation results have been presented to verify the performance.

\section{REFERENCES}

[1] J. Mitola III, "Cognitive radio: an integrated agent architecture for software defined radio," Ph.D. Thesis, KTH Royal Institute of Technology, Stockholm, Sweden, 2000.

[2] D. Cabric, S. M. Mishra, and R. W. Brodersen, " Implementation issues in spectrum sensing for cognitive radios," in Proc. Asilomar Conference on Signals, Systems and Computers, pp. 772-776, Asilomar Grounds, Nov. 2004.

[3] G. Ganesan and Y. Li, "Cooperative spectrum sensing in cognitive radio networks," IEEE Symposium on New Frontiers in Dynamic Spectrum Access Networks (DySPAN'05), pp. 137-143, Bartimore, Nov. 2005.

[4] Z. Han, C. Pandana, and K. J. R. Liu, "Distributive opportunistic spectrum access for cognitive radio using correlated equilibrium and no-regret learning," in Proc. IEEE Wireless Communications and Networking Conference (WCNC 2007), pp. 11-15, Hong Kong, March 2007.

[5] S. Gandhi, C. Buragohain, L. Cao, H. Zheng, and S. Suri, "A general framework for wireless spectrum auctions," IEEE Symposium on New Frontiers in Dynamic Spectrum Access Networks (DySPAN'07), pp. 2233, Dublin, Apr. 2007.

[6] Z. Ji and K. J. R. Liu, "Multi-stage pricing game for collusion-resistant dynamic spectrum allocation," IEEE Journal on Selected Areas in Communications, vol. 26, no. 1, pp. 182-191, Jan. 2008.

[7] Y. Wu, B. Wang, K. J. R. Liu, and T. C. Clancy, "A scalable collusionresistant multi-winner cognitive spectrum auction game," to appear, IEEE Transactions on Communications.

[8] R. Chen, J.-M. Park, and J. H. Reed, "Defense against primary user emulation attacks in cognitive radio networks," IEEE Journal on Selected Areas in Communications, vol. 26, no. 1, pp. 25-37, Jan. 2008.

[9] A. Sethi and T. X. Brown, "Hammer model threat assessment of cognitive radio denial of service attacks," in Proc. IEEE Symposium on New Frontiers in Dynamic Spectrum Access Networks (DySPAN'08), pp.1-12, Chicago, Oct. 2008.

[10] K. Bian and J.-M. Park, "Security vulnerabilities in IEEE 802.22," in Proc. the Fourth International Wireless Internet Conference (WICON 2008), Hawaii, Nov. 2008.

[11] T. C. Clancy and N. Goergen, "Security in cognitive radio networks: threats and mitigation," in Proc. the Third International Conference on Cognitive Radio Oriented Wireless Networks and Communications (CrownCom 2008), pp. 1-8, Singapore, May 2008.

[12] E. Altman, K. Avrachenkov, and A. Garnaev, "A jamming game in wireless networks with transmission cost," in Proc. of NET-COOP 2007. Lecture Notes in Computer Science, vol. 4465, pp. 1-12, 2007.

[13] B. Roberson, "The Colonel Blotto game," Economic Theory, vol. 29, no. 1, pp. 1-24, Sept. 2006.

[14] D. Fudenburg and J. Tirole, Game Theory, MIT Press, 1993. 